\title{
Temperature dependence of the magnon-phonon energy relaxation time in a ferromagnetic insulator
}

\author{
A. I. Bezuglyj, ${ }^{1,2}$ V. A. Shklovskij, ${ }^{2}$ V. V. Kruglyak $\odot,{ }^{3}$ and R. V. Vovk ${ }^{2}$ \\ ${ }^{1}$ National Science Center Kharkov Institute of Physics and Technology, 1. Akademicheskaya Street, Kharkov 61108, Ukraine \\ ${ }^{2}$ Kharkov National University, 4. Svobody Square, Kharkov 61022, Ukraine \\ ${ }^{3}$ School of Physics and Astronomy, University of Exeter, Stocker Road, Exeter EX4 4QL, United Kingdom
}

(Received 13 June 2019; revised manuscript received 19 November 2019; published 9 December 2019)

\begin{abstract}
We have used the Boltzmann kinetic equation for the phonon distribution function to analyze the relaxation kinetics of the spin system of a ferromagnetic insulator $(F)$ lying on a massive dielectric substrate with high thermal conductivity. Under periodic heating of the spin system, the relaxation depends on the thickness of the $F$ layer and on the frequency of the thermal source $\omega$. When the thickness of the F layer is much greater than the phonon-magnon scattering length, the magnon temperature dependence on the frequency has two features related to specific characteristic times of the system. One of them determines the dependence in the low-frequency regime and is related to the average phonon escape time from the F layer to the substrate $\tau_{\text {es }}$. In turn, the highfrequency behavior is determined by the magnon-phonon collisions time $\tau_{m p}$. From the latter, the time of phononmagnon collisions $\tau_{p m}$ can be found. In contrast, the response of effectively thin F layers is characterized by just one feature, which is determined by the time $\tau_{m p}$. Thus, based on the obtained theoretical results, the times $\tau_{\mathrm{es}}$, $\tau_{m p}$, and $\tau_{p m}$ can be deduced from experiments on the parametric excitation of spin waves by electromagnetic radiation modulated at frequency $\omega$.
\end{abstract}

DOI: 10.1103/PhysRevB.100.214409

\section{INTRODUCTION}

Since the late 2000s, spin caloritronics has emerged and is actively developing as a subfield of spintronics. Specifically, spin caloritronics considers the problems of generation and control of spin currents by means of heat fluxes [1-4]. In this area, the spin Seebeck effect (SSE), which consists in generating a spin current by a heat flux, is of great interest [5-13]. The theoretical description of the SSE is based either on the Landau-Lifshitz-Gilbert equation, on the formalism of the kinetic equation, or on the technique of Green functions [14-20]. In these studies, however, the case of stationary heating is considered. At the same time, the problems of nonstationary kinetics of interacting electrons, phonons, and magnons in layered structures used in SSE experiments are also of interest. The relevance of such problems is determined by a number of recent experiments on the observation of SSE under substantially nonstationary conditions of pulsed or periodic heating of the system [21-24].

With nonstationary heating, it is necessary to take into account the processes of energy relaxation. In this paper, we consider the energy relaxation between magnons and phonons, which is interesting from both theoretical and experimental points of view. The relaxation time of magnons on phonons was calculated by A. I. Akhiezer [25] under the assumption that, in a ferromagnet at temperatures much lower than the Curie temperature $T_{C}$, the thermalization time of a magnon gas is much smaller than the time required to reach the equilibrium between magnons and phonons. In Ref. [25], the relaxation problem was considered in the "twotemperature approximation," when the states of magnons and phonons are described by the equilibrium Bose-Einstein functions with temperatures $T_{m}$ and $T_{p}$, respectively. In addition, it was assumed that the magnon temperature is higher than the phonon temperature but the difference in these temperatures is relatively small. Recently, Shklovskij, Mezinova, and Dobrovolskiy [26] considered nonlinear two-temperature relaxation between magnons and phonons in a ferromagnetic insulator, when the temperature of "hot" magnons differs significantly from the temperature of "cold" phonons.

It should be noted, however, that the theoretical calculation of the temperature dependence of the magnon-phonon relaxation times $[25,26]$ is not sufficient in itself, since it is also necessary to indicate a real experimental situation that allows us to determine experimentally these temperature dependencies. Since the ferrodielectric plates (or films) on heat-conducting insulator substrates are usually studied in experiments, for a correct theoretical description, it is also necessary to consider the problem of calculation of the temperature jump at the interface between the ferrodielectric and the insulator (F/I interface). Such a problem of calculating the thermal resistance of the interface $R_{\text {th }}$ (commonly called the "Kapitza resistance") was previously discussed in detail in Ref. [27].

In Ref. [27], it was shown that, at temperatures much lower than the Debye temperature, $\Theta_{D}$, for the nonlinear thermal resistance of the F/I interface, there exists a size effect: The Kapitza resistance for thin $\mathrm{F}$ plates (films) depends on their magnetic properties, whereas for thick $\mathrm{F}$ plates it can be described by the Little formula [28], which does not include the magnetic properties of the ferromagnetic insulator.

Here a linear response of the magnon temperature to periodic heating with frequency $\omega$ is analyzed in the framework of a microscopic approach based on the time-dependent magnon 
temperature $T_{m}(t)$ and the Boltzmann kinetic equation for phonons. This approach is justified at temperatures $T \ll \Theta_{D}$, when, due to the low frequency of phonon-phonon collisions, the phonon temperature approximation is not applicable. As it turns out, the characteristic features of the dependence of the linear responses on the frequency $\omega$ are related to the energy relaxation times of the magnon-phonon system. In this case, account of the phonon exchange between the plate (film) of the ferrodielectric and the massive dielectric substrate with high thermal conductivity makes it possible to include in the microscopic theory the heat-removal effects, which are described in terms of the acoustic impedances of the contacting materials [28]. Thus, our approach gives an analytical description of all the processes associated with the relaxation of heated magnons in a ferrodielectric platedielectric substrate system. Similar problems become relevant for spin caloritronics, where the relationship between the processes of transfer of spins and heat is studied.

The paper has the following structure. The Introduction discusses the formulation of the problem and briefly analyzes the results of other studies associated with it. In Sec. II, the linear response of the magnon temperature to the oscillating heating of magnons is considered in detail. In Sec. III, the temperature dependencies of the frequencies of magnon-phonon and phonon-magnon collisions are calculated analytically and are presented graphically. Section IV contains a discussion of the results and comparison with the experiment. In Sec. V, the conclusions are formulated. The validity of our model is discussed in Appendix.

\section{THE LINEAR RESPONSE OF THE MAGNON TEMPERATURE TO OSCILLATING HEATING}

Under conditions of heating of the magnon subsystem of a ferromagnetic insulator by longitudinal pumping, i.e., by parametric excitation of spin waves, the excess magnon energy is first transferred to the crystal lattice of the ferromagnet and then leaves into the substrate.

When the layer of the ferromagnetic insulator is located on a massive substrate (Fig. 1), the "hot" phonons of the ferromagnet escape to the substrate, and the "cold" phonons of the substrate penetrate into the ferromagnet. The exchange of phonons between the ferromagnet and the substrate is the final stage of relaxation of the excess energy of the magnon system. It is assumed that the substrate is a single crystal with a high thermal conductivity, where the phonons propagate ballistically and do not return to the layer of the ferromagnet. It is assumed that the substrate temperature is constant and equal to $T_{B}$. An important simplifying assumption is the thermalized state of the magnon subsystem, when the magnon distribution function is the Bose-Einstein function with temperature $T_{m}$. We will describe phonons as a yet-unknown distribution function $N_{\mathbf{q}}(z, t)$, which is the occupation number of phonon states with the wave vector $\mathbf{q}$ at time $t$ for a given $z$ coordinate. The axis $z$ is perpendicular to the layer of the ferromagnetic insulator.

The phonon distribution function obeys the Boltzmann equation

$$
\frac{\partial N_{\mathbf{q}}}{\partial t}+s_{m z} \frac{\partial N_{\mathbf{q}}}{\partial z}=I_{p m}\left\{N_{q}, n_{k}\right\}
$$

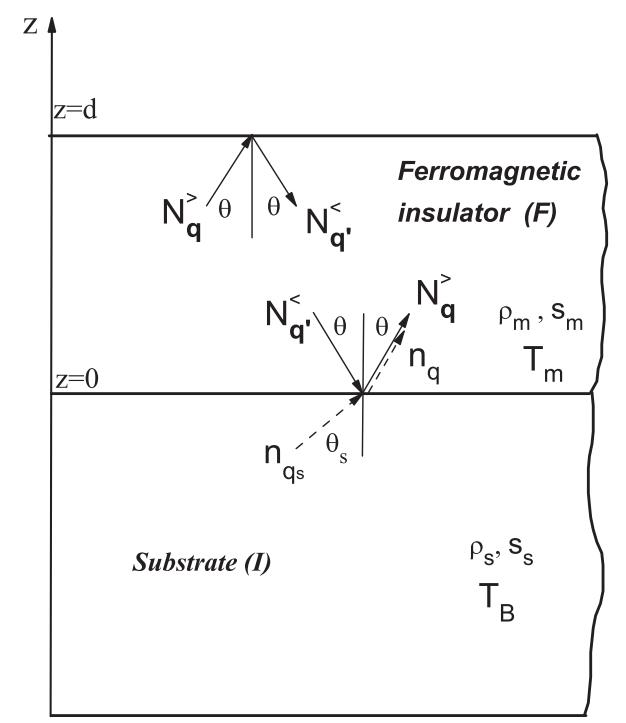

FIG. 1. Refraction and reflection of phonon modes at media boundaries in the layered structure of F/I. The occupation numbers of phonon states with wave vectors $\mathbf{q}$ are denoted by $N_{\mathbf{q}}^{>}\left(N_{\mathbf{q}^{\prime}}^{<}\right)$. The upper index $>(<)$ denotes phonons with a positive (negative) $z$ component of the wave vector. The phonon wave vector with a negative $z$ component is denoted by $\mathbf{q}^{\prime}$. The letters $\rho$ and $s$ denote the densities and speeds of the longitudinal sound of the corresponding media. $T_{B}$ is the temperature of the massive substrate, which plays the role of a thermostat, and $T_{m}$ is the magnon temperature.

Here $n_{k}$ is the magnon distribution function:

$$
n_{k}=\frac{1}{\exp \left(\varepsilon_{k} / T_{m}\right)-1},
$$

where $T_{m}$ is the magnon temperature.

In the long-wavelength limit, which can be used at a low temperature $T_{m} \ll T_{C}$, the magnon dispersion law may be approximated as

$$
\varepsilon_{k}=\Theta_{C}(a k)^{2},
$$

where $\Theta_{C}$ is a temperature that coincides in order of magnitude with the Curie temperature, and $a$ is the lattice constant $\left(k_{B}=1\right)$. The long-wavelength limit corresponds to the strong inequality $a k \ll 1$.

The phonon-magnon collision integral has the form (see Ref. [27])

$$
I_{p m}\left\{N_{\mathbf{q}}\right\}=v_{p m}\left(T_{m}, \mathbf{q}\right)\left[n_{q}\left(T_{m}\right)-N_{\mathbf{q}}(z, t)\right],
$$

where

$$
n_{q}\left(T_{m}\right)=\frac{1}{\exp \left(\hbar \omega_{q} / T_{m}\right)-1}
$$

is the equilibrium (Bose-Einstein) phonon distribution function with temperature $T_{m}$. Here and below, the index $q$ is assigned to phonons, and the index $k$ is assigned to magnons. It is assumed that the temperature of the magnons does not depend on the coordinate $z$ due to the high magnon heat conduction at temperatures much lower than the Debye temperature $\Theta_{D}[29]$.

The frequency of phonon-magnon collisions is given by

$$
v_{p m}\left(T_{m}, q\right)=D\left(T_{m}\right) J_{D}\left(T_{m}, x, y_{0}\right),
$$


where the characteristic frequency is

$$
D\left(T_{m}\right)=\frac{\Theta_{C}\left(T_{m} / \Theta_{C}\right)^{3}}{8 \pi \text { Mas }} .
$$

Here $M$ is the mass of the atoms of the ferromagnetic insulator and $s$ is the average speed of sound. The factor $J_{D}$ is determined by the integral

$$
J_{D}\left(T_{m}, x, y_{0}\right)=\int_{y_{0}}^{\infty} d y(x+y) y\left(\frac{1}{e^{y}-1}-\frac{1}{e^{x+y}-1}\right),
$$

with

$$
y=\frac{\varepsilon_{k}}{T_{m}} ; \quad x=\frac{\hbar \omega_{q}}{T_{m}} ; \quad y_{0}=\frac{\Theta_{D}^{2}}{4 T_{m} \Theta_{C}} .
$$

The magnon temperature $T_{m}$ obeys the heat balance equation for the magnon subsystem

$$
c_{m}\left(T_{m}\right) \frac{d T_{m}}{d t}=W(t)-\frac{1}{d} \int_{0}^{d} d z \int \frac{d^{3} q}{(2 \pi)^{3}} \hbar \omega_{q} I_{p m}\left\{N_{\mathbf{q}}\right\},
$$

where $c_{m}\left(T_{m}\right)$ is the specific heat of magnons and $W(t)$ is the specific power of external sources heating the magnon subsystem. For oscillatory heating (for example, by a highfrequency electromagnetic field) modulated at frequency $\omega$

$$
W(t)=W_{0}+W_{1} e^{-i \omega t} .
$$

In the case of such heating, both the temperature $T_{m}$ and the phonon distribution function also oscillate. For the magnon temperature, we have

$$
T_{m}(t)=T_{m 0}+T_{m 1} e^{-i \omega t},
$$

where the average temperature $T_{m 0}$ is equal to the sum of the temperature of the thermostat and the contribution associated with the stationary heating $W_{0}$. In the linear approximation of interest to us, the oscillating addition to the temperature is small:

$$
\left|T_{m 1}\right| \ll T_{m 0} .
$$

For the phonon distribution function we have

$$
N_{\mathbf{q}}(z, t)=N_{\mathbf{q} 0}(z)+N_{\mathbf{q} 1}(z) e^{-i \omega t} .
$$

In the formulas (11) and (13), $T_{m 1}$ and $N_{\mathbf{q} 1}$ are complex quantities.

The Boltzmann equation for the phonon distribution function takes the following form:

$$
\begin{aligned}
s_{m z} & \frac{d N_{\mathbf{q} 1}}{d z}+\left[v_{p m}\left(T_{m 0}\right)-i \omega\right] N_{\mathbf{q} 1} \\
= & \frac{\partial v_{p m}\left(T_{m 0}, q\right)}{\partial T_{m 0}}\left[n_{q}\left(T_{m 0}\right)-N_{\mathbf{q} 0}(z)\right] T_{m 1} \\
& \quad+v_{p m}\left(T_{m 0}, q\right) \frac{\partial n_{q}\left(T_{m 0}\right)}{\partial T_{m 0}} T_{m 1} .
\end{aligned}
$$

In the case of a weak oscillatory heating, Eq. (9) for $T_{m 1}$ can be linearized

$$
-i \omega c_{m}\left(T_{m 0}\right) T_{m 1}=W_{1}-\frac{1}{d} \int_{0}^{d} d z \int \frac{d^{3} q}{(2 \pi)^{3}} \hbar \omega_{q}\left\{\frac{\partial v_{p m}\left(T_{m 0}, q\right)}{\partial T_{m 0}}\left[n_{q}\left(T_{m 0}\right)-N_{\mathbf{q} 0}(z)\right] T_{m 1}+v_{p m}\left(T_{m_{0}}\right) \frac{\partial n_{q}\left(T_{m 0}\right)}{\partial T_{m 0}} T_{m 1}-v_{p m}\left(T_{m 0}\right) N_{\mathbf{q} 1}(z)\right\} .
$$

Equation (14) and (15) contain a phonon distribution function $N_{\mathbf{q} 0}(z)$, which is a solution of the Boltzmann equation for stationary heating, i.e., in the absence of amplitude modulation of the "heating" electromagnetic radiation. Since the contribution of unmodulated radiation is assumed to be small, then we can replace the average magnon temperature by the temperature of the thermostat: $T_{m 0}=T_{B}$, and $N_{\mathbf{q} 0}=n_{q}\left(T_{B}\right)$. In this case, Eqs. (14) and (15) are simplified:

$$
\begin{aligned}
s_{m z} \frac{d N_{\mathbf{q} 1}}{d z}+\left[v_{p m}\left(T_{B}\right)-i \omega\right] N_{\mathbf{q} 1}=v_{p m}\left(T_{B}\right) \frac{\partial n_{q}\left(T_{B}\right)}{\partial T_{B}} T_{m 1}, & \text { (16) } \\
-i \omega c_{m}\left(T_{B}\right) T_{m 1}= & W_{1}-\frac{1}{d} \int_{0}^{d} d z \int \frac{d^{3} q}{(2 \pi)^{3}} \hbar \omega_{q} \\
\times & {\left[v_{p m}\left(T_{B}\right) \frac{\partial n_{q}\left(T_{B}\right)}{\partial T_{B}} T_{m 1}-v_{p m}\left(T_{B}\right) N_{\mathbf{q} 1}(z)\right] . }
\end{aligned}
$$

The boundary conditions to Eq. (16) are as follows [27]:

$$
\begin{gathered}
N_{\mathbf{q}}^{>}(0, t)=\beta N_{\mathbf{q}^{\prime}}^{<}(0, t)+\alpha n_{q}\left(T_{B}\right), \\
N_{\mathbf{q}}^{>}(d, t)=N_{\mathbf{q}^{\prime}}^{<}(d, t) .
\end{gathered}
$$

Here $\alpha$ is the probability of transmission through, and $\beta=$ $1-\alpha$ is the probability of reflection of the phonon from the interface with the substrate. The condition (18) means that phonons that are specularly reflected from the interface with the substrate and phonons from the substrate that have passed this interface fall into a state with the wave vector $\mathbf{q}$. Note that the condition (18) also assumes that phonons that leave the ferromagnet do not return. Such a pattern is typical for single-crystal substrates with high thermal conductivity and rather narrow layers of a ferromagnet. Equation (19) describes the specular reflection of phonons from the free boundary of the ferromagnet.

In the acoustic mismatch model [28,30,31], the probability $\alpha$ depends on the angle of incidence of the phonon and the acoustic impedances of the F layer and the substrate:

$$
\alpha(\theta)=\frac{4 Z Z_{s} \cos \theta \cos \theta_{s}}{\left(Z \cos \theta_{s}+Z_{s} \cos \theta\right)^{2}} .
$$

Here $Z=\rho_{m} s_{m}\left(Z_{s}=\rho_{s} s_{s}\right)$ is the acoustic impedance of the $\mathrm{F}$ layer (substrate); angles of incidence and refraction are related via $s_{s} \sin \theta=s_{m} \sin \theta_{s}$. Symbols $\rho_{s}$ and $s_{s}$ denote the substrate density and average speed of the longitudinal sound in the substrate. 
In linear order in $W_{1}$, the boundary condition for $z=0$ [Eq. (18)] has the form

$$
N_{\mathbf{q}^{1}}^{>}(0)=\beta N_{\mathbf{q}^{1} 1}^{<}(0),
$$

and on the free surface $z=d$ [Eq. (19)]

$$
N_{\mathbf{q} 1}^{>}(d)=N_{\mathbf{q}^{\prime} 1}^{<}(d) .
$$

When writing the boundary conditions (21) and (22), we took into account that $N_{q 1}(z, t)=N_{q 1}(z) e^{-i \omega t}$.
It is convenient to introduce the notation $v_{p m}\left(T_{B}\right)-i \omega=\tilde{v}$, and $\frac{d n_{q}\left(T_{B}\right)}{d T_{B}}=n_{q}^{\prime}$, since then equations for $N_{\mathbf{q} 1}^{>}$and $N_{\mathbf{q}^{1} 1}^{<}$are simplified:

$$
\begin{gathered}
\left|s_{m z}\right| \frac{d N_{\mathbf{q} 1}^{>}}{d z}+\tilde{v} N_{\mathbf{q} 1}^{>}=v_{p m} n_{q}^{\prime} T_{m 1}, \\
-\left|s_{m z}\right| \frac{d N_{\mathbf{q}^{\prime} 1}^{<}}{d z}+\tilde{v} N_{\mathbf{q}^{\prime} 1}^{<}=v_{p m} n_{q}^{\prime} T_{m 1} .
\end{gathered}
$$

Their solutions are written as

$$
\begin{aligned}
& N_{\mathbf{q} 1}^{>}(z)=\frac{v_{p m} n_{q}^{\prime} T_{m 1}}{\tilde{v}}-\frac{\alpha v_{p m} n_{q}^{\prime} T_{m 1}}{\tilde{v}[1-\beta x(\omega)]} e^{-\tilde{v} z /\left|s_{m z}\right|}, \\
& N_{\mathbf{q}^{\prime} 1}^{<}(z)=\frac{v_{p m} n_{q}^{\prime} T_{m 1}}{\tilde{v}}-\frac{\alpha v_{p m} n_{q}^{\prime} T_{m 1}}{\tilde{v}[1-\beta x(\omega)]} e^{-\tilde{v}(2 d-z) /\left|s_{m z}\right|},
\end{aligned}
$$

where $x(\omega)=\exp \left(-2 \tilde{v} d /\left|s_{m z}\right|\right)$. The substitution of the solutions obtained into (17) gives

$$
\begin{aligned}
-i \omega c_{m}\left(T_{B}\right) T_{m 1}= & W_{1}-\frac{1}{d} \int_{0}^{d} d z \int \frac{d^{3} q}{(2 \pi)^{3}} \hbar \omega_{q} v_{p m} n_{q}^{\prime} T_{m 1}+\frac{1}{d} \int_{0}^{d} d z \int_{q_{z}>0} \frac{d^{3} q}{(2 \pi)^{3}} \hbar \omega_{q} v_{p m}^{2} n_{q}^{\prime} T_{m 1}\left\{\frac{1}{\tilde{v}}-\frac{\alpha}{\tilde{v}[1-\beta x(\omega)]} e^{-\tilde{v} z /\left|s_{m z}\right|}\right\} \\
& +\frac{1}{d} \int_{0}^{d} d z \int_{q_{z}<0} \frac{d^{3} q}{(2 \pi)^{3}} \hbar \omega_{q} v_{p m}^{2} n_{q}^{\prime} T_{m 1}\left\{\frac{1}{\tilde{v}}-\frac{\alpha}{\tilde{v}[1-\beta x(\omega)]} e^{-\tilde{v}(2 d-z) /\left|s_{m z}\right|}\right\} .
\end{aligned}
$$

For $\omega \gg v_{p m}$ in (25), we can neglect the contributions from $N_{\mathbf{q} 1}^{\gtrless}$, since these contributions are proportional to $v_{p m} / \omega$. We arrive at the equation

$$
-i \omega c_{m}\left(T_{B}\right) T_{m 1}=W_{1}-\int \frac{d^{3} q}{(2 \pi)^{3}} \hbar \omega_{q} v_{p m} n_{q}^{\prime} T_{m 1},
$$

which is reduced to

$$
\left[-i \omega c_{m}\left(T_{B}\right)+c_{p}\left(T_{B}\right) \bar{v}_{p m}\left(T_{B}\right)\right] T_{m 1}=W_{1},
$$

where the average frequency of phonon-magnon collisions is given by

$$
\bar{v}_{p m}=\frac{1}{c_{p}\left(T_{B}\right)} \int \frac{d^{3} q}{(2 \pi)^{3}} \hbar \omega_{q} v_{p m}\left(T_{B}, q\right) \frac{\partial n_{q}}{\partial T_{B}}
$$

with the phonon heat capacity

$$
c_{p}\left(T_{B}\right)=\int \frac{d^{3} q}{(2 \pi)^{3}} \hbar \omega_{q} n_{q}^{\prime}\left(T_{B}\right) .
$$

If we introduce the averaged frequency of the magnon-phonon collisions $\bar{v}_{m p}$ according to equation

$$
c_{p}\left(T_{B}\right) \bar{v}_{p m}\left(T_{B}\right)=c_{m}\left(T_{B}\right) \bar{v}_{m p}\left(T_{B}\right),
$$

then we will come to an important result

$$
T_{m 1}=\frac{W_{1}}{c_{m}\left(T_{B}\right)\left[-i \omega+\bar{v}_{m p}\left(T_{B}\right)\right]},
$$

from which it follows that the average frequency of magnonphonon collisions at the temperature $T_{B}$ can be found from the dependence $T_{m 1}(\omega)$ in the high-frequency regime. In the case of low frequencies, when $\omega \ll v_{p m}$, for thick layers of a ferromagnet $(x(0) \ll 1)$, Eq. (17) is reduced to

$$
\begin{aligned}
W_{1}= & -i \omega\left[c_{m}\left(T_{B}\right)+c_{p}\left(T_{B}\right)\right] T_{m_{1}} \\
& +\frac{1}{d} \int_{q_{z}>0} \frac{d^{3} q}{(2 \pi)^{3}} \hbar \omega_{q} v_{p m} \alpha\left|s_{m z}\right| n_{q}^{\prime} T_{m_{1}},
\end{aligned}
$$

where

$$
\frac{1}{d} \int_{q_{z}>0} \frac{d^{3} q}{(2 \pi)^{3}} \hbar \omega_{q} v_{p m} \alpha\left|s_{m z}\right| n_{q}^{\prime}=\frac{\langle\alpha\rangle s}{4 d} c_{p}\left(T_{B}\right)=\frac{c_{p}\left(T_{B}\right)}{\tau_{\mathrm{es}}} .
$$

Here $\tau_{\mathrm{es}}$ is the mean time for phonons to escape from the ferromagnetic insulator to the substrate, and $\langle\alpha\rangle$ is the probability $\alpha$ averaged over the angles of incidence: $\langle\alpha\rangle=$ $\int_{0}^{\pi / 2} \alpha(\theta) \sin (\theta) \cos (\theta) d \theta$.

Now Eq. (31) can be written in a physically transparent form,

$$
-i \omega\left[c_{m}\left(T_{B}\right)+c_{p}\left(T_{B}\right)\right] T_{m 1}+\frac{c_{p}\left(T_{B}\right)}{\tau_{\mathrm{es}}} T_{m 1}=W_{1} .
$$

It follows that the amplitude of the magnon temperature is given by

$$
T_{m 1}=\frac{W_{1}}{\left[c_{m}\left(T_{B}\right)+c_{p}\left(T_{B}\right)\right]\left(-i \omega+1 / \tau_{\mathrm{es}}^{\prime}\right)}
$$

with the renormalized relaxation time

$$
\tau_{\mathrm{es}}^{\prime}=\tau_{\mathrm{es}} \frac{c_{m}\left(T_{B}\right)+c_{p}\left(T_{B}\right)}{c_{p}\left(T_{B}\right)} .
$$

It is seen that, due to the thermal inertia of the magnons, the relaxation time of the magnon temperature is longer than the average phonon escape time from the ferromagnetic insulator $\tau_{\text {es }}$.

In the case of thin $\mathrm{F}$ layers, when $d \ll s / v_{p m}$, the dependence $T_{m 1}(\omega)$ is described by Eq. (30). 


\section{CALCULATION OF THE TEMPERATURE DEPENDENCE OF THE FREQUENCIES OF MAGNON-PHONON AND PHONON-MAGNON COLLISIONS}

If the temperature of the thermostat is renamed as $T$, then Eq. (29) becomes

$$
\bar{v}_{m p}(T)=\frac{1}{c_{m}(T)} c_{p}(T) \bar{v}_{p m}(T),
$$

where the frequency $\bar{v}_{p m}(T)$ is determined by the integral

$$
\bar{v}_{p m}(T)=\frac{1}{c_{p}(T)} \int \frac{d^{3} q}{(2 \pi)^{3}} \hbar \omega_{q} v_{p m}(T, q) \frac{\partial n_{q}}{\partial T}
$$

and Eqs. (6)-(8). The characteristic times of the energy relaxation of phonons on magnons $\tau_{p m}$ and magnons on phonons $\tau_{m p}$ are the inverse of the frequencies $\bar{\nu}_{p m}$ and $\bar{\nu}_{m p}$, respectively. Then

Let us introduce a dimensionless temperature $\bar{t}=T / \Theta_{D}$.

$$
v_{p m}(T)=\tilde{v}_{0}\left(\frac{\Theta_{D}}{\Theta_{C}}\right)^{2} \bar{t}^{3} J_{D}(\bar{t}, q),
$$

where $\tilde{v}_{0}=\Theta_{D} / 8 \pi$ Mas, and the integral $J_{D}(\bar{t}, q)$ is also written in terms of dimensionless values.

In the long-wavelength approximation considered by us, the temperature should be sufficiently low: $T \ll \Theta_{C}$. In addition, in deriving the expression for the phonon-magnon collision frequency, it was assumed that $q a \ll \Theta_{D} / \Theta_{C}$. The last inequality is equivalent to the inequality $T \ll\left(\Theta_{D}\right)^{2} / \Theta_{C}$. From the presented chain of inequalities it follows that $T \ll \Theta_{D}$. This allows us to write down the phonon heat capacity in the form $c_{p}(T)=2 \pi^{2} T^{3} / 15 \hbar^{3} s^{3}$. The expression for $\bar{v}_{p m}(T)$ takes the form of the double integral,

$$
\bar{v}_{p m}=v_{0} \bar{t}^{3} \int_{0}^{\infty} d x \frac{x^{4} e^{x}}{e^{x}-1} \int_{y_{0}}^{\infty} d y \frac{(y+x) y e^{y}}{\left(e^{y}-1\right)\left(e^{x+y}-1\right)},
$$

with the lower limit

$$
y_{0}=\frac{\Theta_{D}}{4 \Theta_{C} \bar{t}}
$$

and the coefficient

$$
\nu_{0}=\tilde{v}_{0} \frac{15}{4 \pi^{4}}\left(\frac{\Theta_{D}}{\Theta}\right)^{2} .
$$

For $T \ll\left(\Theta_{D}\right)^{2} / \Theta_{C}$, the lower limit of the second integral is much greater than unity, whereas the first integral is dominated by $x \lesssim 1$. Using the inequality $y \gg x$, we can calculate these integrals, which gives

$$
\bar{v}_{p m}(\bar{t})=v_{0} \frac{D_{5}}{16}\left(\frac{\Theta_{D}}{\Theta_{C}}\right)^{2} \bar{t} \exp \left(-\frac{\Theta_{D}}{4 \Theta_{C} \bar{t}}\right),
$$

where

$$
D_{5}=\int_{0}^{\infty} d x \frac{x^{4}}{e^{x}-1} \approx 24.88
$$

is a constant. The value $\bar{v}_{p m}^{-1}$ is the characteristic time of energy relaxation of phonons on magnons $\tau_{p m}$. We note that

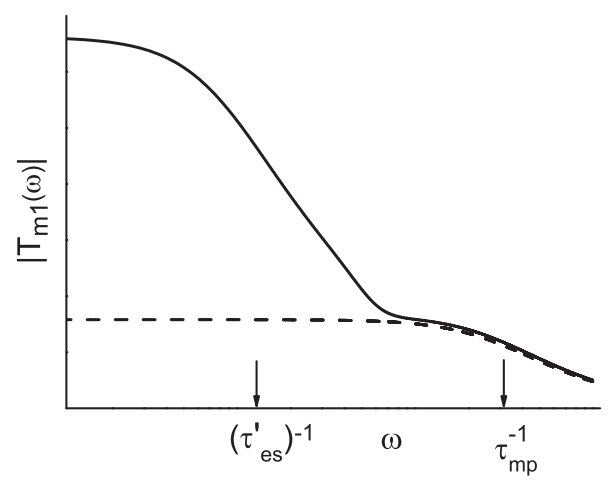

FIG. 2. Schematic dependence of the magnon temperature amplitude on the frequency of the heat source. The solid line corresponds to the effectively thick layer of the ferromagnet, and the dashed line corresponds to the effectively thin layer of the same material. The arrows indicate the frequency at which the oscillation amplitude of the magnon temperature decreases by $\sqrt{2}$ times in comparison with $T_{m 1}(0)$ for both the thick and thin layers of the ferromagnet.

the expression (39) for the frequency of phonon-magnon collisions is consistent (up to numerical multipliers) with the expression for the relaxation time of the phonon temperature obtained in the two-temperature model [25]. form

The phonon specific heat is conveniently written in the

$$
c_{p}(T)=\left(\frac{2 \pi^{2}}{15 a^{3}}\right)\left(\frac{T}{\Theta_{D}}\right)^{3},
$$

while for the magnon heat capacity we obtain

$$
c_{m}(T)=\left(\frac{0.113}{a^{3}}\right)\left(\frac{T}{\Theta_{C}}\right)^{3 / 2} \text {. }
$$

Substitution of these expressions in (35) gives the average frequency of magnon-phonon collisions

$$
\bar{v}_{m p}(\bar{t})=18.2 v_{0}\left(\frac{\Theta_{D}}{\Theta_{C}}\right)^{1 / 2} \bar{t}^{5 / 2} \exp \left(-\frac{\Theta_{D}}{4 \Theta_{C} \bar{t}}\right) .
$$

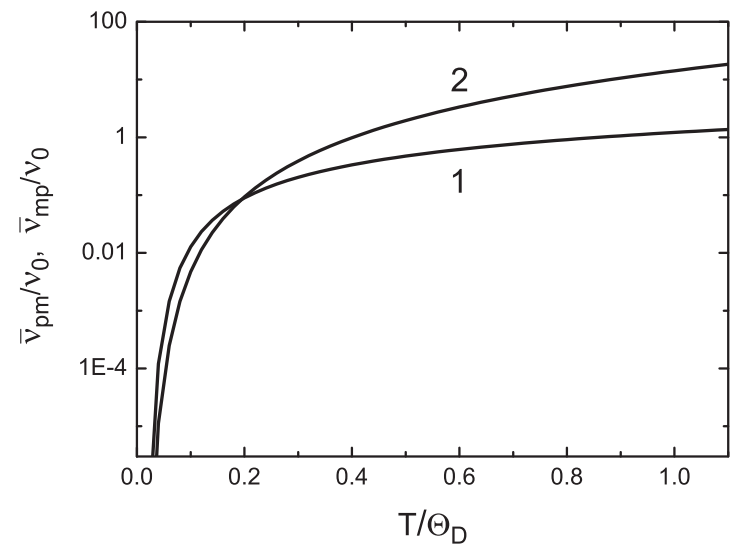

FIG. 3. Temperature dependence of the normalized frequencies of collisions of phonons with magnons (1) and magnons with phonons (2). These dependencies are obtained from the formulas (39) and (40) with $\Theta_{C}=\Theta_{D}$. 
We recall that the value $\left(\bar{v}_{m p}\right)^{-1}$ is equal to the characteristic time of the energy relaxation of magnons on phonons $\tau_{m p}$.

The dependence $\left|T_{m_{1}}(\omega)\right|$ for layers of a ferromagnetic insulator of different thickness follows from Eq. (25). The general form of such dependencies for thick and thin films is shown in Fig. 2. Figure 3 shows the temperature dependence of the frequencies of phonon-magnon and magnon-phonon collisions.

\section{DISCUSSION}

Periodic heating of magnons in layers of yttrium-iron garnet (YIG) of different thickness was carried out in the experiment [21]. In this experiment, the time of magnon-phonon collisions $\tau_{m p}$ was determined by comparing the frequency dependence of the amplitude of oscillations of the difference between the magnon and phonon temperatures $\Delta T_{m p}$ with the dependence $\Delta T_{m p}(\omega) \propto\left[1+\left(\omega \tau_{m p}\right)^{2}\right]^{-1 / 2}$ that follows from the two-temperature model [32]. From Fig. 3 in Ref. [21], it can be seen that for YIG layers with thicknesses $d_{f}>2.7 \times$ $10^{-5} \mathrm{~cm}$, the characteristic frequency $\omega_{3 \mathrm{~dB}}$ is proportional to $d_{f}^{-1}$, where $\omega_{3 \mathrm{~dB}}$ is the frequency at which the amplitude of spin Seebeck voltage $V_{\mathrm{SSE}}(\omega)$ decreases $\sqrt{2}$ times in comparison with $V_{\text {SSE }}(0)$ and which, according to the authors, coincides with $\tau_{m p}^{-1}$. From our analysis [see Eqs. (30), (33), and Fig. 2], it follows that the observed behavior of $\Delta T_{m p}(\omega)$ can be explained by the size effect, when for thick films and low frequencies the value $\omega_{3 \mathrm{~dB}}$ is equal to $\left(\tau_{\mathrm{es}}^{\prime}\right)^{-1}$ and therefore is proportional to $d_{f}^{-1}$. We emphasize that, according to Eq. (30), the frequency $\omega_{3 \mathrm{~dB}}$ coincides with $\tau_{m p}^{-1}$ only in the case of thin layers.

At the same time, we note that a detailed (microscopic) analysis of the experiment in Ref. [21] is a challenge because of the multilayered geometry of the system and a large number of parameters characterizing the kinetics of heat transfer in such a system. A phenomenological analysis of the experiment based on a two-temperature model can be found in Ref. [33].

\section{CONCLUSIONS}

We have shown that the energy relaxation of interacting magnons and phonons in a layer of a ferromagnetic insulator lying on a dielectric substrate with a high thermal conductivity is determined by the following three characteristic times: the mean time for phonons to escape from the ferromagnetic insulator to the substrate, $\tau_{\mathrm{es}}$, the mean scattering time of phonons on magnons, $\tau_{p m}$, and the mean scattering time of magnons on phonons, $\tau_{m p}$. Our results suggest that these times can be found in experiments on periodic heating of the magnetic subsystem of a ferromagnetic insulator, which can be realized by parametric excitation of spin waves by a high-frequency electromagnetic field with a modulated amplitude.

\section{ACKNOWLEDGMENTS}

We thank Professor M. Krawczyk for helpful discussions and support of our work. The research leading to these results has received funding from the European Union's Horizon 2020 research and innovation program under Marie Sklodowska-Curie Grant Agreement No. 644348 (MagIC).

\section{APPENDIX: MODEL VALIDITY}

Let us briefly consider the region of applicability of our model, which is based on the concept of homogeneous magnon temperature. The condition necessary for introducing the magnon temperature is that the magnon-magnon collision frequency is much greater than the collision frequency of magnons with phonons. For four-magnon processes, the average frequency of magnon-magnon collisions is [34]

$$
\bar{v}_{m m} \sim \frac{\Theta_{C}}{\hbar}\left(\frac{T}{\Theta_{C}}\right)^{4} .
$$

A comparison of Eq. (A1) with Eq. (40) shows that, for $\Theta_{D} \gtrsim \Theta_{C}$, the magnon-magnon collision frequency is significantly higher than the collision frequency of magnons with phonons for any $T$. Consequently, the magnon temperature can be introduced at all thermostat temperatures $T_{B}$.

The correction to the temperature of the magnons in the F layer, associated with the finite thermal conductivity of the magnon gas $k_{m}$, is given by the relation $\delta T_{m} \sim Q d / k_{m}$, where $Q$ is the heat flux and $d$ is the thickness of the $\mathrm{F}$ layer. Uniform heating of magnons is associated with the heat flux by the equality $Q=h \Delta T_{m}$, where $h$ is the heat transfer coefficient from the $F$ layer to the substrate. We take into account $\Delta T_{m}$ and omit $\delta T_{m}$, which is justified when $\delta T_{m} \ll \Delta T_{m}$. For YIG, the value of $k_{m}$ was measured in Ref. [35]. The value of $h$ for sufficiently thick F layers can be estimated based on Eq. (11) from the Ref. [27]. (Note that in Ref. [27], the expression for coefficient $A$ in Eq. (11) must contain a numerical factor 120 in the denominator.) The estimate gives $\delta T_{m} / \Delta T_{m} \lesssim 10^{-2} d(\mu \mathrm{m})$ at experiment temperature of $T \lesssim 100 \mathrm{~K}$. With a decrease in temperature $T$, the ratio $\delta T_{m} / \Delta T_{m}$ decreases. The smallness allows us to neglect the inhomogeneity of the magnon temperature in YIG layers with thickness $d \lesssim 10 \mu \mathrm{m}$.

We also note that, in experiments performed on YIG at room temperature, intense pumping led to the chemical potential $\mu_{m} \approx 0.1 \mathrm{~K}$, while the temperature of magnons increased by $\Delta T_{m} \approx 5 \mathrm{~K}$ [36]. Thus, a situation when heating of magnons dominates the chemical potential is quite realistic at room temperature: $\Delta T_{m} \gg \mu_{m}$. With decreasing temperature, the value $\mu_{m}$, being proportional to the product of the temperature $T$ and the time of three-magnon collisions $\tau_{3}$ ( $\tau_{3} \sim T^{-1 / 2}$, see Chap. 7 in Ref. [34]), decreases as $T^{1 / 2}$, and $\Delta T_{m}$, being proportional to the coefficient of heat removal, increases as $T^{-3}$ (Ref. [27]). This allows us to neglect the chemical potential in Eq. (2) at all temperatures below room temperature.
[1] G. E. W. Bauer, A. H. MacDonald, and S. Maekawa, Solid State Commun. 150, 459 (2010).
[2] G. E. W. Bauer, E. Saitoh, and B. J. van Wees, Nat. Mater. 11, 391 (2012). 
[3] G. E. W. Bauer, in Spin Current, edited by S. Maekawa, S. O. Valenzuela, E. Saitoh, and T. Kimura (Oxford University Press, Oxford, UK, 2012).

[4] S. R. Boona, R. C. Myers, and J. P. Heremans, Energy Environ. Sci. 7, 885 (2014).

[5] K. Uchida, S. Takahashi, K. Harii, J. Ieda, W. Koshibae, K. Ando, S. Maekawa, and E. Saitoh, Nature 455, 778 (2008).

[6] K. Uchida, J. Xiao, H. Adachi, J. Ohe, S. Takahashi, J. Ieda, T. Ota, Y. Kajiwara, H. Umezawa, H. Kawai, G. E. W. Bauer, S. Maekawa, and E. Saitoh, Nat. Mater. 9, 894 (2010).

[7] K. Uchida, T. Nonaka, T. Ota, and E. Saitoh, Appl. Phys. Lett. 97, 262504 (2010).

[8] C. M. Jaworski, J. Yang, S. Mack, D. D. Awschalom, J. P. Heremans, and R. C. Myers, Nat. Mater. 9, 898 (2010).

[9] M. Schreier, N. Roschewsky, E. Dobler, S. Meyer, H. Huebl, R. Gross, and S. T. B. Goennenwein, Appl. Phys. Lett. 103, 242404 (2013).

[10] W. X. Wang, S. H. Wang, L. K. Zou, J. W. Cai, Z. G. Sun, and J. R. Sun, Appl. Phys. Lett. 105, 182403 (2014).

[11] B. L. Giles, Z. Yang, J. S. Jamison, and R. C. Myers, Phys. Rev. B 92, 224415 (2015).

[12] E. J. Guo, J. Cramer, A. Kehlberger, C. A. Ferguson, D. A. MacLaren, G. Jakob, and M. Klaui, Phys. Rev. X 6, 031012 (2016).

[13] A. Prakash, B. Flebus, J. Brangham, F. Yang, Y. Tserkovnyak, and J. P. Heremans, Phys. Rev. B 97, 020408(R) (2018).

[14] J. Xiao, G. E. W. Bauer, K.-C. Uchida, E. Saitoh, and S. Maekawa, Phys. Rev. B 81, 214418 (2010).

[15] Steven S.-L. Zhang and S. Zhang, Phys. Rev. B 86, 214424 (2012).

[16] S. M. Rezende, R. L. Rodriguez-Suarez, R. O. Cunha, A. R. Rodrigues, F. L. A. Machado, G. A. Fonseca Guerra, J. C. Lopez Ortiz, and A. Azevedo, Phys. Rev. B 89, 014416 (2014)

[17] M. Schreier, A. Kamra, M. Weiler, J. Xiao, G. E. W. Bauer, R. Gross, and S. T. B. Goennenwein, Phys. Rev. B 88, 094410 (2013).
[18] Y. H. Shen, X. S. Wang, and X. R. Wang, Phys. Rev. B 94, 014403 (2016).

[19] H. Adachi, J. I. Ohe, S. Takahashi, and S. Maekawa, Phys. Rev. B 83, 094410 (2011).

[20] H. Adachi, K. Uchida, E. Saitoh, and S. Maekawa, Rep. Prog. Phys. 76, 036501 (2013).

[21] M. Schreier, F. Kramer, H. Huebl, S. Geprags, R. Gross, S. T. B. Goennenwein, T. Noack, T. Langner, A. A. Serga, B. Hillebrands, and V. I. Vasyuchka, Phys. Rev. B 93, 224430 (2016).

[22] J. Kimling, G.-M. Choi, J. T. Brangham, T. Matalla-Wagner, T. Huebner, T. Kuschel, F. Yang, and D. G. Cahill, Phys. Rev. Lett. 118, 057201 (2017).

[23] T. Hioki, R. Iguchi, Zh. Qiu, D. Hou, K.-I. Uchida, and E. Saitoh, Appl. Phys. Express 10, 073002 (2017).

[24] A. Fognini, T. U. Michlmayr, A. Vaterlaus, and Y. Acremann, J. Phys.: Condens. Matter 29, 214002 (2017).

[25] A. Akhieser, J. Phys. (USSR) 10, 217 (1946).

[26] V. A. Shklovskij, V. V. Mezinova, and O. V. Dobrovolskiy, Phys. Rev. B 98, 104405 (2018).

[27] V. A. Shklovskij, V. V. Kruglyak, R. V. Vovk, and O. V. Dobrovolskiy, Phys. Rev. B 98, 224403 (2018).

[28] A. W. Little, Can. J. Phys. 37, 334 (1959).

[29] A. I. Akhiezer and L. A. Shishkin, Zh. Eksp. Teor. Fiz. 34, 1267 (1958) [Sov. Phys. JETP 34, 875 (1958)].

[30] E. T. Swartz and R. O. Pohl, Rev. Mod. Phys. 61, 605 (1989).

[31] S. B. Kaplan, J. Low Temp. Phys. 37, 343 (1979).

[32] D. J. Sanders and D. Walton, Phys. Rev. B 15, 1489 (1977).

[33] Y. Xu, W. Zhao, and S. Mangin, Phys. Rev. B 98, 144408 (2018).

[34] A. I. Akhiezer, V. G. Bar'yakhtar, and S. V. Peletminskii, Spin Waves (North-Holland, Amsterdam, 1968).

[35] S. R. Boona and J. P. Heremans, Phys. Rev. B 90, 064421 (2014).

[36] C. Du, T. van der Sar, T. X. Zhou, P. Upadhyaya, F. Casola, H. Zhang, M. C. Onbasli, C. A. Ross, R. L. Walsworth, Ya. Tserkovnyak, and A. Yacoby, Science 357, 195 (2017). 